\title{
Clinical and Imaging Improvement After the Atlasprofilax Method in a Patient with Cervicobrachial Syndrome and Temporomandibular Joint Disorders. A Case Report
}

\section{José G León ${ }^{1}$, Lluís Manent ${ }^{3}$, Kathleen Lewis ${ }^{4}$ and Orlando Angulo ${ }^{2 *}$}

${ }^{1}$ Department of Physical Activity and Sports Medicine, San José Children's Hospital, Bogotá DC, Colombia

${ }^{2}$ Professor and Researcher at Universidad Cooperativa de Colombia, Villavicencio and Professor at the Department of Continued Education, Fundacion Universitaria de Ciencias de la Salud, Bogotá DC, Colombia

${ }^{3}$ Professor in Agreement at the Department of Continued Education, Fundacion

Universitaria de Ciencias de la Salud, Bogotá DC, Colombia

${ }^{4}$ Independent Researcher, California, USA

*Corresponding Author: Orlando Angulo, Professor and Researcher at Universidad Cooperativa de Colombia, Villavicencio and Professor at the Department of Continued Education, Fundacion Universitaria de Ciencias de la Salud, Bogotá DC, Colombia.
Received: August 30, 2021

Published: September 24, 2021

(C) All rights are reserved by Orlando Angulo., et al.

\begin{abstract}
A 30-year-old woman with chronic shoulder pain, cervicobrachial syndrome with functional motor loss and paresthesia, carpal tunnel syndrome, tension-type headache, and temporomandibular disorders was referred for a computed tomography scan to evaluate a possible atlantoaxial rotatory subluxation and asymmetry of the lateral atlantodental interval prior to the application of the Atlasprofilax method. The same tomographic study was performed after the application of this method. Normalization of the atlantoaxial rotatory subluxation and lateral atlantodental interval was observed post-treatment, as well as substantial improvement in the patient's symptoms. Normalization of previous midline deviation and better alignment of C3-C4 spinous processes was also visualized after the application of the treatment. We also discuss the probable relationship, relevance, and implications of clinical or subclinical atlantoaxial rotatory subluxations and lateral atlantodental interval. In addition, we consider the fascia and suboccipital muscles that could be underlying abnormalities at the craniocervical junction and affecting proximal or distal regions. The effectiveness of the Atlasprofilax method as an innovative, safe, and non-invasive approach is preliminarily postulated and recommended to be the subject of further consideration and clinical studies..
\end{abstract}

Keywords: Atlantoaxial Rotatory Subluxation; Lateral Atlantodental Interval; Odontoid-Lateral Mass Interspaces Asymmetry; Atlasprofilax; Mechanotransduction; Craniocervical Joint; Fascia; Vertebral Subluxation; Suboccipital Muscles

\section{Introduction and Case Report}

A 30-year-old woman with diagnosed chronic shoulder pain, cervicobrachial syndrome with functional motor loss and paresthesia, carpal tunnel syndrome, deviation of dental midline, tension-type headache, and temporomandibular disorders was referred for a computed tomography scan to examine a possible atlantoaxial rotatory subluxation (ARS) and asymmetry of the lateral atlantodental interval (LADI) prior to the application of the Atlasprofilax method and to asses probable clinical improvement in her ailments. The same tomographic study was performed after the ap- 
plication of this method. An additional photographic examination of midline deviation was performed. The aim of this examination was to determine if the Atlasprofilax method could produce not only beneficial improvement in the described clinical symptoms, but also better symmetry in the LADI and ARS and better alignment in the dental midline and C3-C4 spinous processes by means of CT imaging.

\section{Methods, measurements, and endpoints}

Imaging examination: methods and measurements

2D and 3D computed tomography imaging was used by means of an i-CAT Gendex CB-500 HD 3D CBCT 14×8 FOV/FLX V10 device. The patient's chin and head were immobilized with the device stabilizers, and 2D/3D images were taken pre- and post-application of the Atlasprofilax method.

To measure and determine ARS, the rotation angle in degrees of C1 was measured in the axial plane by drawing a line from each transverse process of the atlas before and after Atlasprofilax treatment, taking the horizontal basal tomographic line as a reference. In the coronal plane, the degree of symmetry of the lateral masses of the Atlas and the transverse processes before and after the application of the Atlasprofilax method was taken as a reference according to the morphological visualization of these structures. To determine the LADI, the left vs. right difference of the atlanto-odontoid space was measured in the axial plane by drawing vertical lines from same references point (most inner border of $\mathrm{C} 2$ and most inner border of C1's lateral mass) before and after the application of the Atlasprofilax method. This same measurement was also applied to the 3D image of the computed tomographic reconstruction from the anterior coronal plane and the superior plane.

To measure the deviation of the spinous processes of $\mathrm{C} 3-\mathrm{C} 4$, the same tomograph was used in a posterosuperior image, and a straight line was drawn from the same odontoid landmark to C5 to determine changes in the alignment of these spinous processes before and after therapy.

For photographic examination of the midline deviation, the patient was placed in a seated position without a backrest. She maintained her natural posture, with her feet resting on the floor and her knees at a 90-degree angle. A Dine Corp. PENTAX Optio M40 camera of 8.0 megapixels and 3x optical zoom, $6.3 \mathrm{~mm}-18.3 \mathrm{~mm}$, was placed on an adjustable tripod at the patient's mouth height at a distance of $33 \mathrm{~cm}$. A Bimler correlometer was used for the photographic analysis of the midline, as it has a vertical reference line and one perpendicular to it for the horizontal measurement in millimeters. The distance between the upper and lower dental midlines was measured both at maximum intercuspation and at maximum opening.

\section{Clinical examination: Methods and measurements}

Visual Analogue Scale (VAS) was clinically used to measure pain intensity before and after the therapy (in two controls at 1 month and 8 months) in headache, pain associated with cervicobrachial syndrome and TMJ-associated jaw pain.

\section{Objectives: Imaging and clinical endpoints}

The objective of the study had two purposes: observing imaging improvement in ARS/LADI and clinical improvements in the described symptoms.

At the imaging level, variations in the rotation of $\mathrm{C} 1$ in the axial plane were taken as endpoints, as well as changes in the coronal visualization and presentation of the morphological structures of C1 (lateral masses of the Atlas and its transverse processes) towards a better symmetry after treatment. The vertical alignment of the C3-C4 spinous processes in relationship with the odontoid was the endpoint to compare findings on the improvement of symmetry and alignment of these structures before and after therapy. The endpoint of the dental midline deviation was the observation of its symmetry in occlusion and maximum opening before and after treatment.

At the clinical level, the differential result of pain according to VAS for headache, shoulder pain associated with cervicobrachial syndrome and the absence or presence of motor alterations and paresthesias also associated with cervicobrachial syndrome were taken as endpoints.

\section{Intervention}

The intervention consisted of a non-invasive neurostimulation with a special device that used controlled percussion vibropressure at special frequency with an adapted head that was applied during eight minutes on several key points of the suboccipital area to stimulate certain muscle and fascial receptors aiming at a deep mechanotransduction effect on the suboccipital muscles. 
Clinical and Imaging Improvement After the Atlasprofilax Method in a Patient with Cervicobrachial Syndrome and Temporomandibular Joint Disorders. A Case Report

Results (Imaging findings and clinical findings)

Imaging findings

Atlas rotation and transverse processes

On examining the axial plane in 2D prior to the application of the Atlasprofilax method, we observe that the patient presents with the atlas rotated 8 . The right transverse process of the atlas is anterior in comparison to the left transverse process, which is consistent with ARS (Figure 0). This rotation means that in the coronal view the right lateral mass is visible, but not the right transverse process (Figure 0).

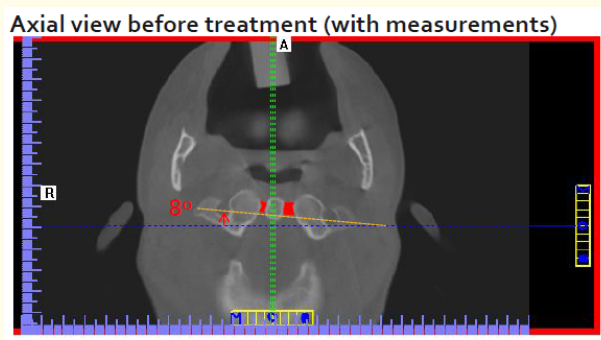

Axial view before treatment (without measurements)

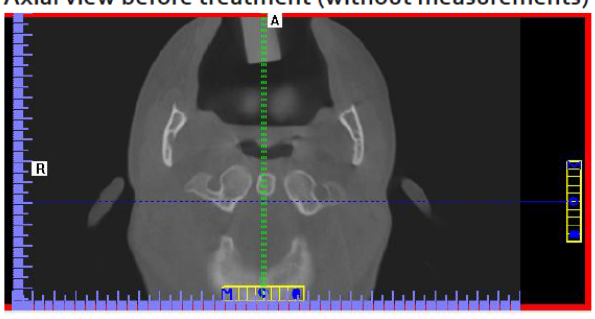

Coronal view before treatment (with measurements)

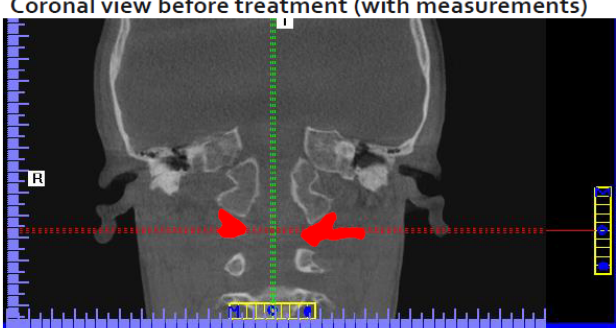

Coronal view before treatment (without measurements)

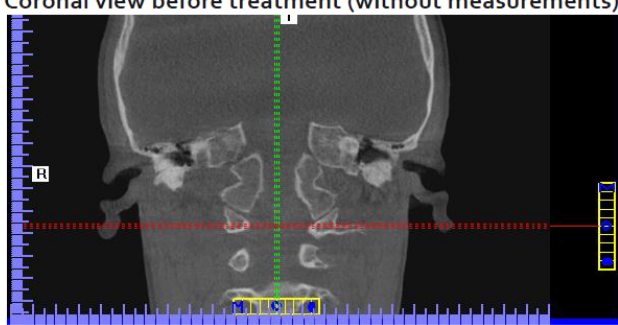

Figure 0: 2D axial and coronal views before the application of the Atlasprofilax method. In the axial view (left), the right transverse process of the atlas is rotated anteriorly $8^{\circ}$. In the coronal view (right), the right transverse process is not visualized, due to the $\mathrm{C} 1$ rotation. Both lateral masses and the left transverse process are visualized.

After the application of the Atlasprofilax method, both transverse processes of the atlas exhibit a symmetry (Figure 1) that is not present on the pre-treatment image. Rotation of $\mathrm{C} 1$ is no longer visible in the axial view. In the coronal plane, both the lateral masses of the atlas and the transverse processes appear symmetrical (Figure 1).

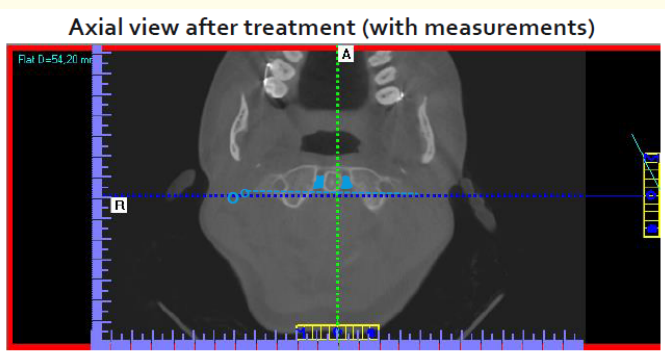

Coronal view after treatment (with measurements)
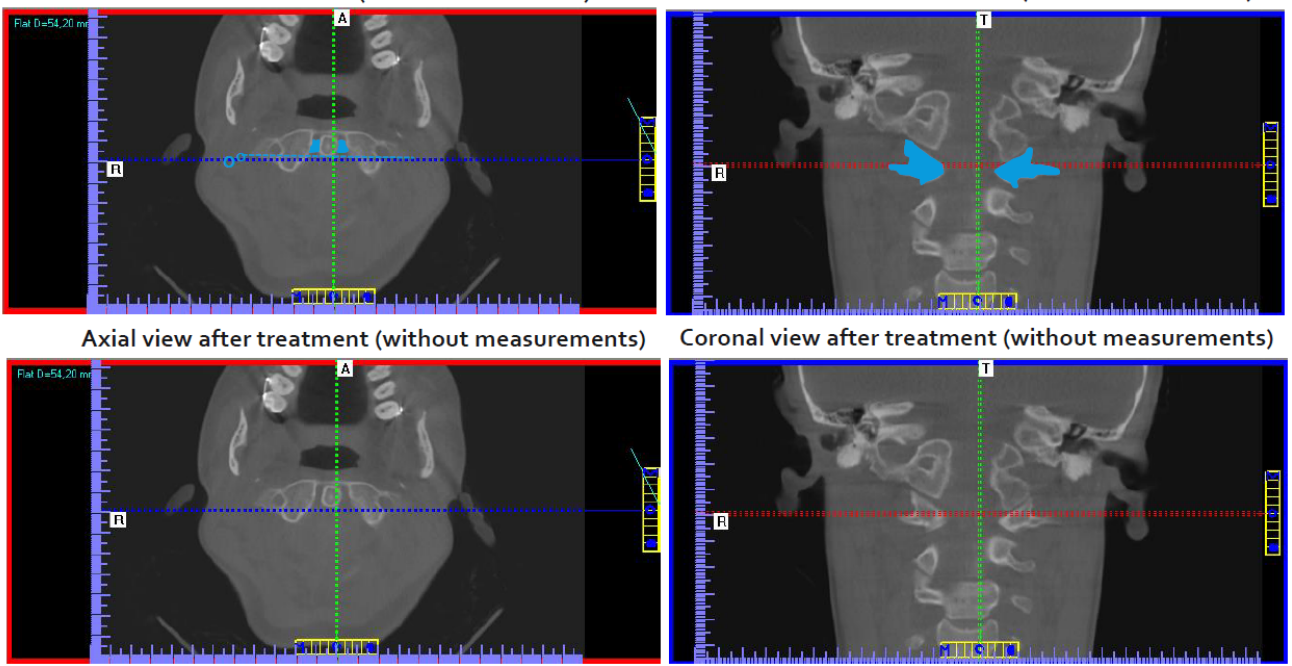

Figure 1: 2D axial plane (left) and coronal plane (right) views of atlas after application of the Atlasprofilax method. The ARS is resolved, with 0 o of rotation (left) and a symmetrical presentation of C1's lateral masses (right). 


\section{LADI}

On examination of the axial plane in 2D prior to the application of the Atlasprofilax method, it is observed that the patient presents with an asymmetrical LADI, in which the left LADI is approximately double the right LADI (Figure 2).
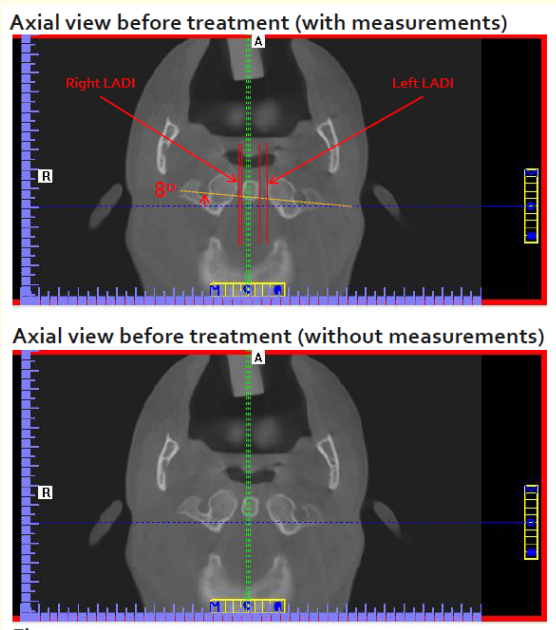

After the application of the Atlasprofilax method, the 2D axial plane view shows that the left LADI is almost equal to the right LADI (Figure 2).

A more symmetrical LADI was confirmed through 3D reconstruction (Figure 3).

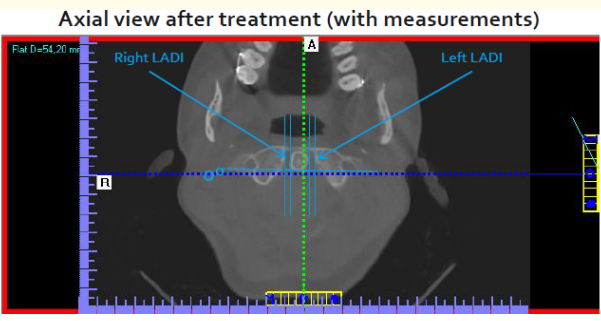

Axial view after treatment (without measurements)

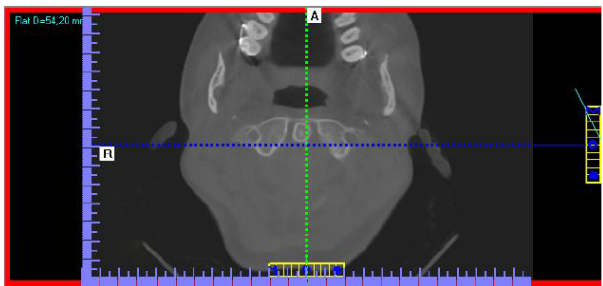

Figure 2: LADI pre-treatment (left), showing the left LADI space is approximately double the right LADI (shown in red). The LADI posttreatment (right) is almost perfectly

symmetrical (shown in blue).

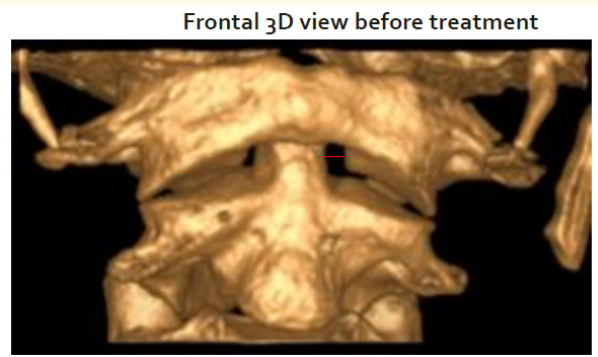

Upper ${ }_{3}$ D view before treatment

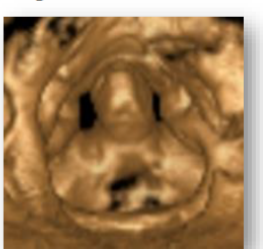

Frontal ${ }_{3} \mathrm{D}$ view after treatment

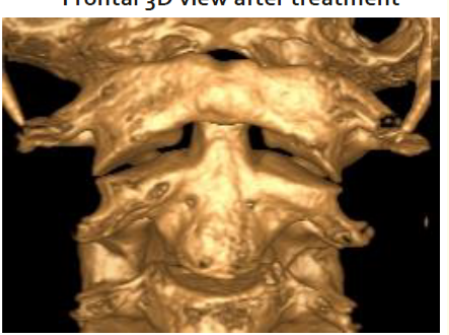

Upper ${ }_{3} \mathrm{D}$ view after treatment

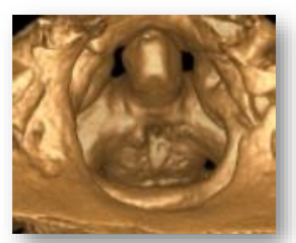

Figure 3: 3D reconstruction. Frontal LADI view with

asymmetry before the treatment (left upper image) and LADI improvement after the treament (right upper image). 3D LADI's upper view with asymmetry before the treatment (left lower image) and 3D LADI's upper view showing better symmetry after the treatment (right lower image). 
Clinical and Imaging Improvement After the Atlasprofilax Method in a Patient with Cervicobrachial Syndrome and Temporomandibular Joint Disorders. A Case Report

\section{Dental midline deviation}

Deviation from the dental midline has been correlated in several studies with temporomandibular joint disorders (TMJD) and has been proposed as a predictor of these disorders [1]. An interaction between suboccipital muscles and temporomandibular joint (TMJ) muscles also is described in the literature [2].

Since the patient came to the maxillofacial dentistry and orthodontics clinic due to symptoms related to TMJD, the position of the dental midline was checked before and after the application of the Atlasprofilax method, as well as one-month post-application. The frontal photographs, taken with closed mouth at maximum inter- cuspation and open mouth at maximum opening, demonstrate a dental midline deviation of approximately $1 \mathrm{~mm}$ before the application of the therapy and a total normalization immediately after the therapy. This improvement is maintained at one month, as seen in a supplemental image. Improvement of the maximum mouth opening was noted immediately after the therapy and was even greater one month post-therapy (Figure 4).

Improvement in the alignment of the $\mathrm{C} 3-\mathrm{C} 4$ spinous processes

Additionally, an improvement in the alignment of the spinous processes of C 3 and C4 was observed before and after the Atlasprofilax therapy (Figure 5).

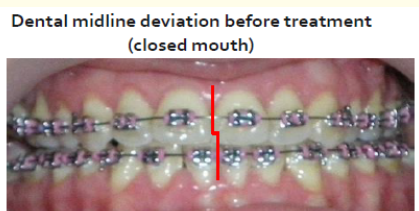

Dental midline deviation beforetreatment

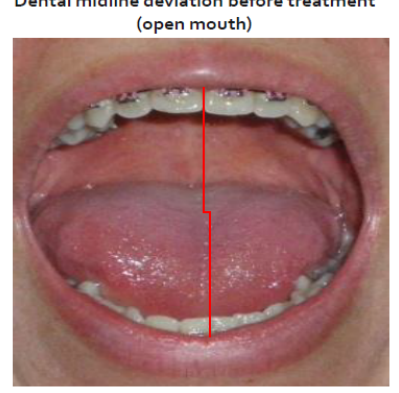

Dental midline deviation right after the treatment (closed mouth)

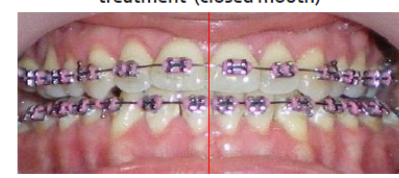

Dental midline deviation right after the treatment (open mouth)

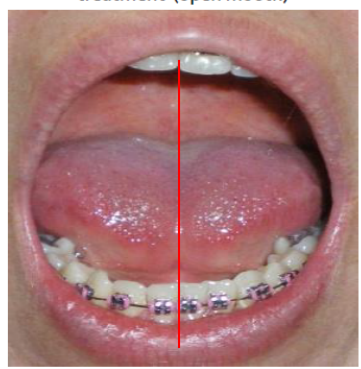

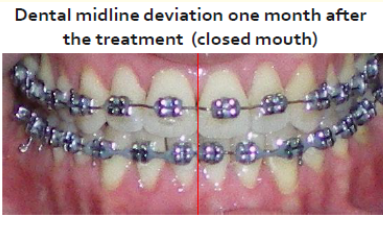

Dental midline deviation one month after the treatment (open mouth)

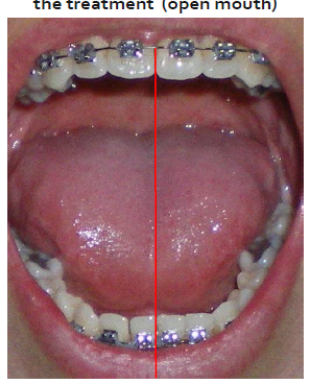

Figure 4: Frontal photographic view of dental midline

deviation before the treament (left), inmediately after (center), and one month post-treatment (right).

Upper $3^{D}$ view before treatment including $C_{3}$ $\mathrm{C}_{4}$ spinous processes alignment

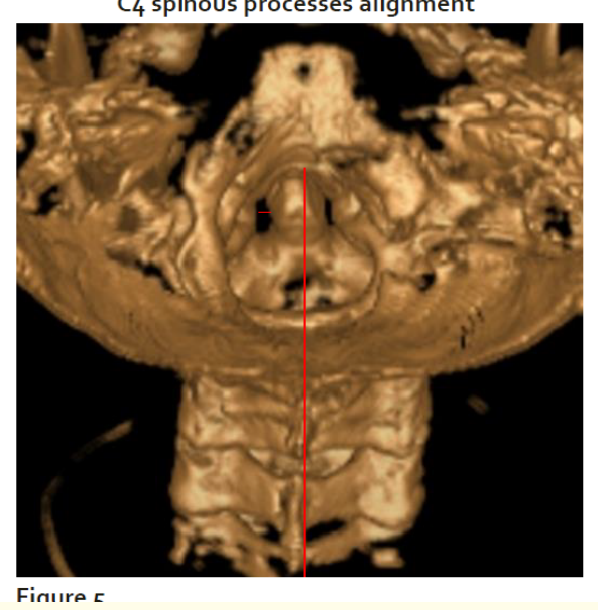

Upper ${ }_{3} D$ view after treatment including $C_{3}-C_{4}$ spinous processes alignment

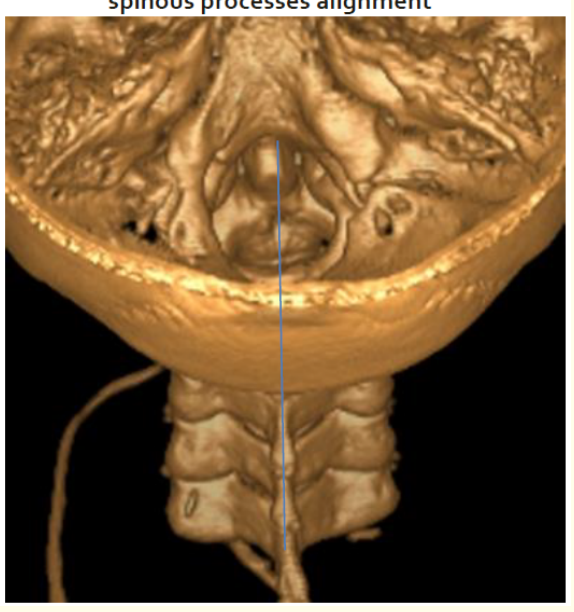

Figure 5: Upper-posterior image of skull, odontoid and part of axis vertebra and C3-C4 spinous processes (Left = before the treatment; right $=$ immediately after the treatment). 
Clinical and Imaging Improvement After the Atlasprofilax Method in a Patient with Cervicobrachial Syndrome and Temporomandibular Joint Disorders. A Case Report

\section{Clinical findings}

According to post-treatment clinical examination and the patient's reporting, all TMJ- associated symptoms such as chewing problems, joint crepitation and joint locking at maximum opening disappeared, including jaw pain. Persistent shoulder pain decreased from 9 VAS prior to the therapy down to 2 after it on VAS (See table 1). Paresthesia and motor loss in the affected right upper limb consistent with carpal tunnel syndrome and cervicobrachial syndrome disappeared after application of the Atlasprofilax method (See table 1). The patient also reported a substantial improvement in recurrence and intensity of chronic tension headaches (See table 1). These clinical improvements measured at one month in the clinical control remained unchanged in several consecutive supplementary controls over a period of 8 months.

\begin{tabular}{|l|c|c|c|}
\hline Symptom & $\begin{array}{c}\text { VAS prior } \\
\text { therapy }\end{array}$ & $\begin{array}{c}\text { VAS 1-month } \\
\text { after therapy }\end{array}$ & $\begin{array}{c}\text { VAS } \\
\text { 8-months } \\
\text { after therapy }\end{array}$ \\
\hline Headache & 8 & 2 & 2 \\
\hline $\begin{array}{l}\text { Upper limb pain } \\
\text { associated to } \\
\text { cervicobrachial } \\
\text { syndrome }\end{array}$ & 9 & 2 & 2 \\
\hline $\begin{array}{l}\text { Jaw pain associated } \\
\text { to TMJ disorders }\end{array}$ & 7 & 0 & 0 \\
\hline $\begin{array}{l}\text { Presence } \\
\text { before } \\
\text { therapy }\end{array}$ & $\begin{array}{c}\text { 1-month after } \\
\text { therapy }\end{array}$ & $\begin{array}{c}\text { 8-months } \\
\text { after therapy }\end{array}$ \\
\hline $\begin{array}{l}\text { Upper limb } \\
\text { paresthesia }\end{array}$ & Yes & No & No \\
\hline $\begin{array}{l}\text { Upper limb motor } \\
\text { loss }\end{array}$ & Yes & No & No \\
\hline
\end{tabular}

Table 1: Evolution of symptoms before and after therapy in two controls at one month and at eight months.

\section{Abbreviations}

ARS: Atlantoaxial Rotatory Subluxation; CCJ: Craniocervical Joint; CT: Computed Tomography; ECM: Extracellular Matrix; LADI: Lateral Atlantodental Interval; NAARS: Non-Acute Atlantoaxial Rotatory Subluxation; TMJ: Temporomandibular Joint; TMJD: Temporomandibular Joint Disorder; VAS: Visual Analogue Scale.

\section{Discussion and Conclusion}

Atlantoaxial rotatory subluxation: An ongoing debate

Acute atlantoaxial rotatory subluxations have been extensively studied, mainly in the context of severe post-traumatic cervical injury, non-traumatic spontaneous arousal [3], Grisel's syndrome, Down Syndrome, rheumatoid arthritis or Marfan Disease. These subluxations can be due to the development of deformational changes in the bony anatomy of the atlantoaxial joint. The associated symptoms can vary from torticollis to chronic neck pain and headache to various neurological symptoms that may be persistent [4]. In the most extreme cases, they can be the result of fractures in the lower cervical spine, humerus and clavicle; or fractures in the head and neck region, such as Jefferson's C1 fracture, or odontoid fractures, that may require surgical intervention.

Non-acute atlantoaxial rotatory subluxation (NAARS) and clinical or subclinical atlantoaxial rotatory subluxations (ARS) have been the subject of very little study by physicians and radiologists, remaining the object of study of other disciplines such as chiropractic or osteopathy. The chiropractic definition of a vertebral subluxation differs from a medical allopathic vertebral subluxation, according to the WHO [5]. The disparity in definitions and therapeutic approaches poses a problem when trying to medically elucidate their relevance and clinical significance-a significance which still is poorly understood by physicians.

Chiropractors developed definitions, such as the Vertebral Subluxation Complex [6], to describe such disorders that are non-acute but seem to have some clinical relevance. There is some evidence of clinical and biological relevance for the vertebral subluxation problem as a subclinical manifestation that could be underestimated $[7,8]$. Even if there is not a clear consensus among chiropractors [9] on the vertebral subluxation complex, some evidence indicates that there might be associations with pain and disability [10]. The different approaches and definitions of chiropractic and medicine on that subject pose a problem when trying to unify concepts, establish its clinical relevance and develop therapeutic treatments $[10,11]$. There are several radiological studies on NAARS/ARS $[12,13]$ and several treatments to fix this misalignment that might support the consideration of ARS/NAARS and rotatory craniocervical joint (CCJ) dysfunction as significant health issues. Metabolic abnormalities in the soft tissues surrounding the CCJ may have im- 
plications in ARS etiology, pathophysiology and clinical presentation that may have been understudied.

Asymmetry of the lateral atlantodental interval: A controversial issue

There is an ongoing scientific debate regarding the clinical relevance of LADI asymmetry in healthy subjects [14-16]. Some authors consider this asymmetry to be a normal common variant [17], while others consider it to be a subclinical sign for a rotatory condition (C0-C1-C2) with clinical implications. Atlantoaxial traumatic injury has been linked to ARS [18], but there is also radiographic evidence of ARS in healthy patients without symptoms, leaving open to debate the question of whether ARS is of no clinical significance, an unrecognized subclinical sign in various clinical manifestations or an indicator predicting future onset of clinical manifestations.

A study concluded that ARS in children without cervical tenderness and abnormal atlantodental interval was not significant [19]. Another study concluded that "[A]symmetry of the LADI is not unusual in asymptomatic children" [20]. One study found that $54 \%$ of non-injured patients had LADI asymmetries [14], concluding that LADI asymmetry is also a normal condition in non-injured subjects. As the focus of this issue has been the bony structures, which are well-visualized in x-rays and computed tomography, analysis of the soft structures of the CCJ has not received as much attention. The CCJ is composed also of a large variety of soft tissue structures, such as suboccipital muscles, ligaments, and fascia, with a wide range of metabolic processes related to the extracellular matrix (ECM), mechanoreceptors, nociceptors, and many other cell types. Therefore, the answer to the question as to whether ARS can be diagnosed solely by means of x-rays and CT remains partially unresolved. One theory is that soft tissue abnormalities at the CCJ level could express a subclinical ARS/NAARS that is not diagnosable, due to the inability to determine soft tissue changes at a histological level.

Could atlantoaxial rotatory subluxation be the basis of ailments such as tension-type headache, cervicobrachial syndrome, carpal tunnel syndrome or TMJ-associated disorders?

Subclinical abnormalities at the CCJ level could lead to mechanical and metabolic alterations of muscle and fascial continuum, affecting proximal regions such as the TMJ and distal regions such as the neck and the upper limb. Posture can be affected as a result.
Poor posture is seen in the Upper Crossed Syndrome. The Upper Crossed Syndrome, which may lead to dysfunction at the CCJ region, has been associated with musculoskeletal pain, cervicogenic tension-type headache [21] and fibromyalgia [22].

Suboccipital muscles play a very important role in the biomechanics of the CCJ [23] and in proprioception and nociception [24]. Evidence of morphological abnormalities in the rectus capitis major (RCM) and rectus capitis minor (RCMi) have been found in several pathological conditions [25-31]. The RCM, RCMi and the obliquus capitis inferior also connect to the cervical dura mater via a fascial membrane called the myodural bridge [32]. Abnormal suboccipital muscle tension or failure of the myodural bridge's function to prevent dural infolding have been proposed as mechanisms for chronic neck pain, headaches, and other ailments [33].

Chronic neck and head pain [25] have been correlated with abnormalities in the suboccipital region, including neurogenic atrophy [26] and muscle replacement by fatty infiltration [27], especially in whiplash-associated disorders [27], chronic tension headache and migraine $[25,30]$.

Neck pain and back pain often occur with fibromyalgia [34], which is a condition that overlaps with cervical myofascial pain syndrome [35]. Sixty-three patients suffering from fibromyalgia who were treated with the Atlasprofilax method obtained very significant and long-lasting reductions in VAS pain. Six months after the procedure, they had improved to the extent that they no longer met the American College of Rheumatology's fibromyalgia classification and criteria [36].

Another study of 151 patients treated with the same Atlasprofilax method showed significant results in the amelioration of TMJD-related symptoms [37]. TMJD is a disorder that has been linked to suboccipital muscle abnormalities [2].

When looking at the probable pathophysiology of cervicobrachial syndrome, it can be observed that the suboccipital muscles connected to the CCJ can have an impact not only in the upper cervical spine, but also in the lower cervical spine and the upper thoracic spine. For example, consider the levator scapula, which attaches to the C1-C4 transverse processes and forms part of the floor of the posterior triangle, through which crosses the brachial plexus. Abnormal tone of the suboccipital muscles and/or fascial deformation could affect the levator scapula and could be one mechanism 
responsible for cervicobrachial symptoms through connections with the Cruveilhier plexus [38]. Pain, mechanical dysfunction and limitation of upper-limb movement are also related to abnormalities of the cervical spine muscles [39]. Origination of these problems could be in the suboccipital muscles and involve subclinical ARS that may progress to clinical manifestations.

There is growing scientific evidence that the fascial network has a role in distally spreading CCJ-related abnormalities. The skull, CCJ and its suboccipital muscles are interlinked with the deep fascia of the neck. The deep cervical fascia connects the ligamentum nuchae and spinous processes and fully encloses the vertebrae [40]. Fascia directly connects to (and cannot be separated from) joints, organs, muscles, tendons and meninges. It is an integral part of body biomechanics [41].

Fascia acts metabolically and biomechanically as a tridimensional network with specific functions in viscera, in permeating blood vessels, and in allowing proper nerve mobility. Fascia connects directly to the dura and other meninges (which are fascial structures). Fascia also supports muscle chains in providing joint mobility and enhancing muscular function, playing an important role in proprioception, interoception [42] and nociception. In this context, fascial deformities and abnormalities affecting the extracellular matrix (ECM) and fascial metabolism [43] could lead to movement restrictions, pain, body and joint imbalance and impairment of nerves, vessels, and joints.

The fascial continuum and the body's information exchange rely on ECM scaffolds consisting of protein (e.g. collagen), glycoproteins, and glycosaminoglycans. ECM-to-cell binding and interaction is dependent on the exchange of information with surface cell receptors of the cytoskeleton. Some surface cell receptors, such as integrins, connect the ECM to the cytoskeleton. They respond to mechanical signals from the ECM and transmit the signal to the cytoskeleton. This process of mechanotransduction results in changes in cellular biochemistry [43].

The surrounding environment plays an important role in cell survival, since cellular capacity to receive continuous information can regulate future behavior within the ECM. This is because some signaling pathways related to compression, stress, tension and liquids shear rely on monitoring of mechanical information coming from the ECM [44].
The concept of fascintegrity considers solids and liquids [45] as a single continuum that transmits and receives mechano-metabolic information in real time, affecting entire body functioning. Thus, the microvacuolar fascia can send mechanical stimuli to other parts, thanks to its entropic constitution [46]. Trying to separate macroscopic biomechanics from microscopic metabolic cell activity in the cytoskeleton is not possible. Cells recognize overstretching, deformation, hysteresis, and entanglement dynamically. This results in an adaptation in the cytoskeleton which leads to realignment. When mechanical forces with certain frequencies are exerted on the fascia, a modification in the cytoskeleton can occur [46].

Although research is limited as to the efficacy of myofascial therapy [47], several manual myofascial treatments appear to successfully treat musculoskeletal and pain disorders [48]. Some suboccipital myofascial inhibition techniques at the CCJ are useful to alleviate pain and increase mobility, but their effect is mostly shortterm and they must be repeated [49]. A plausible explanation on why such temporarily successful fascial treatments are not so effective long-term could be the difficulty in achieving and inducing long-lasting metabolic changes in the suboccipital muscles and deep neck fascia solely by using manual mobilization techniques.

The Atlasprofilax method, an innovative approach for explaining and treating ARS and LADI asymmetries, is in alignment with this fascintegrity approach. It involves the use of a specific vibropressure device and well-determined protocols that can generate, thanks to mechanotransduction, long-lasting metabolic changes in soft tissue structures of the CCJ region. These changes can be transferred by the fascia to distal regions. The device and its protocols target deep myofascial tissues that can be better treated by creating a so-called "bio-piezoresistive effect", leading to longer-lasting results than those commonly observed in manual fascial therapy.

In this manner, long-term or permanent improvement in stressed proteins and negative entropic biomechanics of fascial membranes, which had previously resulted in entangled and abnormal fascial patterns, could potentially be achieved. The CCJ and its fascial and deep muscle structures are designed to protect critical structures in the upper neck, such as the bulbo-medullary junction and the brainstem. Due to these highly protective functions, the CCJ, fascia and deep muscles may be resistant to 
external manual stimuli, and therefore do not easily change their metabolic and structural conformation. Thus, ARS or NAARS may involve abnormal patterns in the fascial biotensegrity (that are not visible by means of x-rays nor CT imaging), leading over time to changes in metabolism and mechanical alteration of CCJ soft and bony structures. In this way, a vicious circle is established: when mechanics are permanently affected, cell dysfunction can increase due to mechanotransduction. And vice versa. C0-C1 mobility is well protected and restricted (especially in rotation), thanks to various protective mechanisms that involve the proprioceptive functions of suboccipital muscles which prevent dural infolding and spinal cord impingement. These protective functions probably are why attempts to restore normal CCJ, fascial biotensegrity and muscle function through chiropractic or osteopathic manipulation do not exhibit long-lasting results. Other segments of the body have been more successfully treated with manual therapeutics because they don't have the same overprotective mechanisms that exist for the $\mathrm{CCJ}$ region. In the Atlasprofilax approach, a non-manual discontinuous vibropressure through specific frequencies and applied angles to CCJ soft structures could regulate fascial and muscles abnormalities at a mechanical and metabolic level as well. This way, fascial anisotropy could be harmonized, having a positive effect on the stress and interactions of discontinuous forces between hard tissue compression elements such as CCJ bony structures and elastic tension elements such as fascia and muscles. Thus, the overprotective fascial ability of self-regulation at the highly protected CCJ segment could be more easily treated and rehabilitated, thanks to the enhancement that the Atlasprofilax method induces specifically at this segment though mechanotransduction and bio-piezoresistive effects.

This innovative and non-invasive approach could shed some light onto the problem of the clinical relevance of ARS and LADI asymmetry in healthy and nonhealthy subjects. Currently accepted norms for ARS and LADI may presage future clinical manifestations. If the Atlasprofilax method results in improved symmetry (as demonstrated in this case report), we may need to narrow the range of normal values for ARS, NAARS and LADI.

\section{Teaching Point}

This report highlights the importance of LADI and ARS asymmetry (even within the ranges usually accepted for "normal asymmetry") that could be the basis of clinical manifestations and ailments, such as those described in this case. The Atlasprofilax method concentrates on normalizing myofascial structures at the structural and metabolic level, which are associated with TMJD and probably with other skeletal muscle problems. Application of this method could lead, as is observed in this case, to greater symmetry of upper cervical bony structures, a more equal LADI and improved ARS. This case report broadens the debate on the relevance of these problems. It preliminarily offers a potentially effective treatment of several LADI/ARS-related ailments that involve understudied metabolic fascial functions, suboccipital muscles and other soft structures in the CCJ region.

\section{Authors' Contributions}

José G. León: Conceptualization, Supervision; Orlando Angulo: reviewing, editing, supervision; Lluís Manent: Writing - original draft, review and editing, supervision; Kathleen Lewis: editing, supervision \& review.

\section{Acknowledgements}

We want to posthumously thank Dr. Victoria Gutiérrez Navas $\dagger$ for her contribution to this work.

\section{Disclosures}

José G. León: Medical director of the Atlasprofilax Academy Switzerland Latin America Corp.

Orlando Angulo: Reports no declaration of interest.

Lluis Manent: Director of the Atlasprofilax Academy Switzerland Latin America corp.

Kathleen Lewis: Practitioner of the Atlasprofilax Method.

\section{Consent}

Written informed consent from the participant subject was obtained.

\section{Human and Animal Rights}

As an observational case report, no experiments were performed on human subjects. Nevertheless; this case study is within the ethical standards of the Helsinki Declaration of 1975, as revised in 2000 . 


\section{Bibliography}

1. Almăşan OC., et al. "Skeletal pattern in subjects with temporomandibular joint disorders". Archives of Medical Science 9.1 (2013): 118-126.

2. Assche R Van., et al. "Interaction between Suboccipital Muscles and TMJ Muscles" (2006).

3. Brunner S and Rovsing H. "Spontaneous Subluxation of the Atlanto-Axial Joint”. Acta Pædiatrica 51.1 (1962): 88-93.

4. AW S. "Subluxation of the atlanto-axial joint, sequel to inflammatory processes of the neck". The Journal of Pediatrics 35.4 (1949): 451-464.

5. WHO. WHO guidelines on basic training and safety in chiropractic. Eff Br mindfulness Interv acute pain Exp An Exam Individ Differences 1 (2005): 1699.

6. Watkins RJ. "Subluxation terminology since 1746". The Journal of the Canadian Chiropractic Association (1968): 18-24.

7. Evans DK. "Anterior cervical subluxation". Journal of Bone and Joint Surgery - Series B 58.3 (1976): 318-321.

8. Rome PL. "Usage of chiropractic terminology in the literature: 296 ways to say "subluxation": complex issues of the vertebral subluxation". Tech Chiropractic 8.2 (1996): 49-60.

9. Keating JC., et al. "Subluxation: dogma or science?" Chiropractic and Osteopathy 13.1 (2005): 17.

10. Dishman R. "Review of the literature supporting a scientific basis for the chiropractic subluxation complex". The Journal of Manipulative and Physiological Therapeutics 8.3 (1985): 163174.

11. Kent C. "Models of Vertebral Subluxation: A Review". Trauma 1.1 (1996): 1-7.

12. AEBJAMTCP. "Diagnostic Imaging Practice Guidelines for Musculoskeletal Complaints in Adults-An Evidence-Based Approach-Part 3: Spinal Disorders". Journal of Manipulative and Physiological Therapeutics 31.1 (2008): 33-88.

13. Hubbard TA., et al. "Inter- and intraexaminer reliability of the Blair protractoview method: examination of a chiropractic radiographic technique". Journal of Chiropractic Medicine 9.2 (2010): 60-68.
14. Lee S., et al. "Asymmetry of the odontoid-lateral mass interspaces: A radiographic finding of questionable clinical significance". Annals of Emergency Medicine 15.10 (1986): 11731176.

15. Harty JA., et al. "Odontoid lateral mass asymmetry: Do we over-investigate?" Emergency Medicine Journal 22.9 (2005): 625-627.

16. Borders HL., et al. "Pediatric lateral atlantodental interval: How much asymmetry is normal?" Journal of Computer Assisted Tomography 35.5 (2011): 557-559.

17. Chen Y., et al. "A three-dimensional study of the atlantodental interval in a normal Chinese population using reformatted computed tomography". Surgical and Radiologic Anatomy 33.9 (2011): 801-806.

18. Billmann F., et al. "Occurrence and significance of odontoid lateral mass interspace asymmetry in trauma patients". World Journal of Surgery 37.8 (2013): 1988-1995.

19. A EDM Y. "Asymmetry of the odontoid lateral mass interval in pediatric trauma CT: Do we need to investigate further?" The American Journal of Neuroradiology 37.4 (2016): 176-179.

20. Mendenhall SK., et al. "Evaluation of lateral atlantodental interval asymmetry in the pediatric age group: normative values". Journal of Neurosurgery: Pediatrics 22.2 (2018): 195-199.

21. MKM. "Upper crossed syndrome and its relationship to cervicogenic headache". The Journal of Manipulative and Physiological Therapeutics 27.6 (2004): 414-420.

22. KDJLAKSDMRMBFBH. "Postural control deficits in people with fibromyalgia: A pilot study". Arthritis Research and Therapy 13.4 (2011).

23. Hallgren RC and Rowan JJ. "Implied evidence of the functional role of the rectus capitis posterior muscles". The Journal of the American Osteopathic Association 120.6 (2020): 395-403.

24. McPartland JM and Brodeur RR. "Rectus capitis posterior minor: A small but important suboccipital muscle". Journal of Bodywork and Movement Therapies 3.1 (1999): 30-35.

25. Fernández-De-Las-Peñas C., et al. "Association of cross-sectional area of the rectus capitis posterior minor muscle with active trigger points in chronic tension-type headache: A pilot

Citation: Orlando Angulo., et al. "Clinical and Imaging Improvement After the Atlasprofilax Method in a Patient with Cervicobrachial Syndrome and Temporomandibular Joint Disorders. A Case Report". Acta Scientific Orthopaedics 4.10 (2021): 92-102. 
Clinical and Imaging Improvement After the Atlasprofilax Method in a Patient with Cervicobrachial Syndrome and Temporomandibular Joint Disorders. A Case Report

study". American Journal of Physical Medicine and Rehabilitation 87.3 (2008): 197-203.

26. Andary MT., et al. "Neurogenic atrophy of suboccipital muscles after a cervical injury". American Journal of Physical Medicine and Rehabilitation 77.6 (1998): 545-549.

27. Elliott J., et al. "Fatty infiltration in the cervical extensor muscles in persistent whiplash-associated disorders: A magnetic resonance imaging analysis". Spine 31.22 (2006): 847-855.

28. Rick Hallgren. "Magnetic Resonance Imaging of the Upper Cervical Spine. Detection of Atrophic Changes in Rectus Capitis Posterior Minor Muscles". Chiropr Ressour Organ.

29. Elliott JM., et al. "Differential changes in muscle composition exist in traumatic and nontraumatic neck pain". Spine 39.1 (2014): 39-47.

30. Yuan XY., et al. "Correlation between chronic headaches and the rectus capitis posterior minor muscle: A comparative analysis of cross-sectional trail". Cephalalgia 37.11 (2017): 10511056.

31. Fernández-De-Las-Peñas C., et al. "Myofascial trigger points and their relationship to headache clinical parameters in chronic tension-type headache". Headache 46.8 (2006): 12641272.

32. Enix DE., et al. "The cervical myodural bridge, a review of literature and clinical implications". The Journal of the Canadian Chiropractic Association 58.2 (2014): 184-192.

33. Hallgren RC., et al. "Clinical implications of a cervical myodural bridge" (1997).

34. Yunus MB. "The prevalence of fibromyalgia in other chronic pain conditions". Pain Research and Treatment (2012).

35. CakitCakit BD., et al. "Comorbidity of fibromyalgia and cervical myofascial pain syndrome". Clinical Rheumatology 29.4 (2010): 405-411.

36. Malagón J., et al. "A Therapeutic Alternative in the Management of Fibromyalgia". Rev Cuarzo 23.1 (2017): 30.

37. Gutiérrez Navas VE. “Efecto De La Terapia Atlasprofilax ${ }^{\circledR}$ Sobre Los Síntomas Relacionados Con Disfunción Temporomandibular, Bruxismo Y La Relación De Las Líneas Medias Dentales. Ustasalud 12.2 (2013): 124.

38. Tubbs RS., et al. “Cruveilhier plexus: An anatomical study and a potential cause of failed treatments for occipital neuralgia and muscular and facet denervation procedures: Laboratory investigation". Journal of Neurosurgery 115.5 (2011): 929-933.

39. O'leary S., et al. "Muscle dysfunction in cervical spine pain: Implications for assessment and management". Journal of Orthopaedic and Sports Physical Therapy 39.5 (2009): 324-333.

40. Sutcliffe P and Lasrado S. "Anatomy, Head and Neck, Deep Cervical Neck Fascia (2020).

41. Schleip R., et al. "Fascia: The Tensional Network of the Human Body: The science and clinical applications in manual and movement therapy". Fascia: The Tensional Network of the Human Body (2012): 1-535.

42. Cathcart E., et al. "Immediate biomechanical, systemic, and interoceptive effects of myofascial release on the thoracic spine: A randomised controlled trial". Journal of Bodywork and Movement Therapies 23.1 (2019): 74-81.

43. Wang HQ., et al. "The effect on the extracellular matrix of the deep fascia in response to leg lengthening". BMC Musculoskeletal Disorders (2008): 9.

44. Cheng B., et al. "Cellular mechanosensing of the biophysical microenvironment: A review of mathematical models of biophysical regulation of cell responses". Physics of Life Reviews (2017): 88-119.

45. Bordoni B and Myers T. "A Review of the Theoretical Fascial Models: Biotensegrity, Fascintegrity, and Myofascial Chains". Cureus (2020).

46. Bordoni B., et al. "Biotensegrity or Fascintegrity?" Cureus (2019).

47. McKenney K., et al. "Myofascial release as a treatment for orthopaedic conditions: A systematic review". The Journal of Athletic Training 48.4 (2013): 522-527.

48. MSA., et al. "Effectiveness of myofascial release: Systematic review of randomized controlled trials". Journal of Bodywork and Movement Therapies 19.1 (2015): 102-112.

49. Jiang W., et al. "Effectiveness of physical therapy on the suboccipital area of patients with tension-type headache". Medicine 98.19 (2019): e15487.

\section{Volume 4 Issue 10 October 2021}

(C) All rights are reserved by Orlando Angulo., et al. 